\title{
Associations of MTA1 expression with CT features, pathology and prognosis of elderly patients with non-small cell lung cancer
}

\author{
NING YANG ${ }^{1 *}$, CHUANMING LI $^{2 *}$, XIAO HAN ${ }^{3}$, ZHIHUA FENG $^{4}$, \\ FENG QIU ${ }^{5}$ and JUNQING HAN ${ }^{1}$
}

\begin{abstract}
${ }^{1}$ Tumor Research and Therapy Center, Shandong Provincial Hospital, Cheeloo College of Medicine, Shandong University, Jinan, Shandong 250021; ${ }^{2}$ Department of Pain Management, Jiading Hospital of Traditional Chinese Medicine, Shanghai 201800; ${ }^{3}$ Department of Experiment, Tumor Hospital Affiliated to Guangxi Medical University, Nanning, Guangxi 530021; ${ }^{4}$ Department of Proctology, Jiading Hospital of Traditional Chinese Medicine, Shanghai 201800; ${ }^{5}$ Department of Pain Management, Shandong Provincial Hospital Affiliated to Shandong First Medical University, Jinan, Shandong 250021, P.R. China
\end{abstract}

Received December 20, 2019; Accepted June 30, 2020

DOI: $10.3892 / 01.2020 .12034$

\begin{abstract}
Associations of metastasis-associated protein 1 (MTA1) expression with computed tomography (CT) features, pathology and prognosis of elderly patients with non-small cell lung cancer (NSCLC), and its clinical significance were explored. A total of 98 elderly patients with NSCLC were selected and underwent CT examination. The expression of MTA1 in carcinoma tissues and para-carcinoma normal tissues was detected via immunohistochemistry, and its associations with CT features, pathology and prognosis were analyzed. The results manifested that the expression of MTA1 in carcinoma tissues was significantly higher than that in para-carcinoma normal tissues, and it was associated with the degree of differentiation, stage and lymph node metastasis $(\mathrm{P}<0.05)$. Besides, the high expression of MTA1 was also related to the spicule sign, pleural indentation and lymph node metastasis $(\mathrm{P}<0.05)$ as well as the CT perfusion parameter capillary permeability (PMB) $(\mathrm{P}<0.05)$, but not to blood volume (BV), blood flow (BF) or time to peak (TTP). Moreover, the patients with high expression of MTA1 had significantly shorter survival time and a remarkably lower 5-year survival rate than those with low expression of MTA1 $(\mathrm{P}<0.05)$. In conclusion, MTA1 plays a certain role in the occurrence and development of NSCLC in elderly patients and has an association with their
\end{abstract}

Correspondence to: Professor Junqing Han, Tumor Research and Therapy Center, Shandong Provincial Hospital, Cheeloo College of Medicine, Shandong University, 324 Jingwuweiqi Road, Jinan, Shandong 250021, P.R. China

E-mail: feiruo19760812@163.com

*Contributed equally

Key words: computed tomography, non-small cell lung cancer in the elderly, metastasis-associated protein 1 , prognosis prognosis, which can provide references for the treatment and prognosis of NSCLC, with important clinical significance.

\section{Introduction}

Lung cancer is the most common malignant tumor. The morbidity and mortality rates rank first among all malignant tumors, seriously threatening human health (1). Lung cancer includes small cell lung cancer (SCLC) and non-small cell lung cancer (NSCLC), the latter of which is dominant (2). The early clinical symptoms of NSCLC are atypical, so the patients are in the middle and advanced stage once diagnosed, with unsatisfactory efficacy and poor prognosis. In recent years, the efficacy of specific gene- or protein-targeted therapies for middle and advanced NSCLC has greatly improved. However, the prognosis of NSCLC is still very poor and the 5 -year survival rate has not improved. Therefore, study on the prognostic factors of NSCLC would be helpful for effective judgment of the prognosis and the formulation of individualized treatment plan (3). Metastasis is the main cause of death of patients $(4,5)$. Metastasis-associated protein 1 (MTA1) plays an important role in tumor metastasis and often serves as a potential indicator for malignant potential in clinic (6). Some studies have reported that MTA1 protein expression is associated with invasiveness, metastasis and clinical outcome in patients with NSCLC (7-9). Computed tomography (CT) examination is a conventional examination method for NSCLC, which can fully reflect the pathophysiological and molecular biological characteristics of tumors, and it has important value in guiding the diagnosis and therapeutic evaluation $(10,11)$. However, the relationship between MTA1 protein expression and CT features has not been investigated so far. It was hypothesized that there was some correlation between the expression of MTA1 protein and CT features. Therefore, actively exploring the associations between the imaging features of NSCLC and molecular markers can provide a reliable theoretical basis for targeted therapy of NSCLC. 


\section{Patients and methods}

General data. A total of 98 elderly patients with NSCLC treated in Shandong Provincial Hospital (Shandong, China) from July 2012 to June 2014 were selected. Inclusion criteria: i) Patients meeting the diagnostic criteria for NSCLC, ii) those undergoing CT examination, and iiii) those without mental disorders and receiving no surgery and chemoradiotherapy. Exclusion criteria: i) Patients with failure of major organs, such as heart, liver and kidney, ii) those with an allergic history of contrast agent, or iii) those who underwent drug therapy or surgical therapy previously (Table I).

The study was approved by the Ethics Committee of Shandong Provincial Hospital. Written informed consents were obtained from the patients and/or guardians.

CT examination. Before the examination, all patients underwent solid fasting for $6 \mathrm{~h}$, and they were informed of relevant precautions. The patients removed any metal objects, raised both hands to place them on the both sides of the headrest. The dual-source CT machine (Siemens AG) was used for plain scan (tube voltage: $120 \mathrm{kV}$, rotation time: $0.5 \mathrm{sec}$, field of view: $414 \mathrm{~mm}$, and slice thickness: $5 \mathrm{~mm}$ ) from apex of the lung to costophrenic angle of patients under a supine position. Then the patients drank properly, and were injected with $50 \mathrm{ml}$ of iohexol $(350 \mathrm{mg} / \mathrm{ml})$ at a rate of $6 \mathrm{ml} / \mathrm{s} \mathrm{using}$ the automatic high-pressure injector also under the supine position. After injection for $7 \mathrm{sec}$, the perfusion scan was performed once per second for a total of 31 times under BODY PCT mode (tube current: $110 \mathrm{mAs}$, matrix: 512x512, collimation: $2 \times 32 \times 1.2 \mathrm{~mm}$, rotation time: $0.28 \mathrm{sec}$, and tube voltage: $80 \mathrm{kV}$ ). Image analysis and processing: The scanning images were transferred to the processing workstation, and the clear perfusion slice with less moving artifact was analyzed and processed by software. Finally, the blood flow $(\mathrm{BF})$, blood volume (BV), permeability surface (PS) and mean transit time (MTT) were calculated.

Detection of MTA1 expression. The expression of MTA1 in lung tissue specimens was determined using immunohistochemistry. The paraffin-embedded tissues were sliced into $4 \mu \mathrm{m}$-thick sections using a microtome (Leica Microsystems $\mathrm{GmbH}$ ), baked at $60^{\circ} \mathrm{C}$ for $2 \mathrm{~h}$, deparaffinized with xylene and soaked in gradient ethanol and distilled water for $5 \mathrm{~min}$. Thereafter, the sections were added with $50 \mu \mathrm{l}$ of $3 \%$ hydrogen peroxide solution, and incubated at $20^{\circ} \mathrm{C}$ for $10 \mathrm{~min}$ to block the activity of endogenous peroxidase. After washing with phosphate buffered saline (PBS) 3 times ( $5 \mathrm{~min} / \mathrm{time}$ ), the sections were incubated with $50 \mu \mathrm{l}$ of primary antibodies MTA1 (1:300; cat. no. ab71153; Abcam) at $4^{\circ} \mathrm{C}$ overnight, and then incubated again with secondary antibodies (1:500; cat. no. ab7090; Abcam) at $20^{\circ} \mathrm{C}$ for 10 min, followed by color development using diaminobenzidine (DAB) kit (Beijing ZSGB Biotechnology Co., Ltd.), hematoxylin counterstaining for $2 \mathrm{~min}$ and sealing with neutral balsam.

Evaluation of indexes. Two senior radiologists conducted the imaging analysis. CT perfusion parameters included BV, BF, capillary permeability (PMB) and time to peak (TTP).
Table I. General data of patients.

\begin{tabular}{lc}
\hline Characteristics & Patients \\
\hline Total no. & 98 \\
Mean age (years) & $68.74 \pm 5.86$ \\
Sex (male/female) & $69 / 29$ \\
Tissue type [n (\%)] & \\
Squamous carcinoma & $53(54.08)$ \\
Adenocarcinoma & $45(45.92)$ \\
Clinical stage [n (\%)] & \\
I-II & $34(34.69)$ \\
III-IV & $64(65.31)$ \\
\hline
\end{tabular}

Expression of MTA1 in lung tissues determined using immunohistochemistry. Ten fields of view (magnification, x200) were randomly selected in each section to detect the percentage of positive cells (brown yellow cells), and the percentage point (PP) was given: i) 0 point: no positive cells; ii) 1 point: percentage of positive cells $<5 \%$; iii) 2 points: $>5 \%$ to $\leq 20 \%$ positive cells; iv) 3 points: percentage of positive cells $>20 \%$. Besides, the staining intensity (SI) was also scored: i) 0 point: no staining; ii) 1 point: light yellow; iii) 2 points: brown yellow; iv) 3 points: dark brown. Finally, the immunoreactivity score (IRS) was calculated: IRS $=$ PPxSI. IRS $>4$ points indicates high expression, while IRS $\leq 4$ points indicates low expression (12).

Statistical analysis. Statistical Product and Service Solutions (SPSS) 19.0 software (SPSS Inc., Chicago, IL, USA) was used for data processing. Measurement data were expressed as mean \pm standard deviation (mean $\pm \mathrm{SD}$ ), and t-test was performed. Enumeration data were expressed as case (n) or percentage (\%), and $\chi^{2}$ test was performed. $\mathrm{P}<0.05$ indicates a statistically significant difference.

\section{Results}

Expression of MTA1 in carcinoma tissues and para-carcinoma normal tissues. As shown by immunohistochemistry in Fig. 1, the positive expression rate of MTA1 in 98 patients was $60.20 \%$ (59/98), and MTA1 was mainly expressed in the nucleus of carcinoma tissues. The positive expression of MTA1 was higher in poorly differentiated carcinoma than in moderately differentiated and well differentiated carcinoma, and the MTA1 expression was very low in para-carcinoma normal tissues.

Association between MTA1 and clinicopathological features of patients. The expression of MTA1 was associated with the degree of differentiation, stage and lymph node metastasis $(\mathrm{P}<0.05)$, but not with the age, sex or tissue type $(\mathrm{P}>0.05)$ (Table II).

Association between CT image features and MTAl expression. The expression of MTA1 was related to the spicule sign, pleural indentation sign and lymph node metastasis sign $(\mathrm{P}<0.05)$ (Table III). 


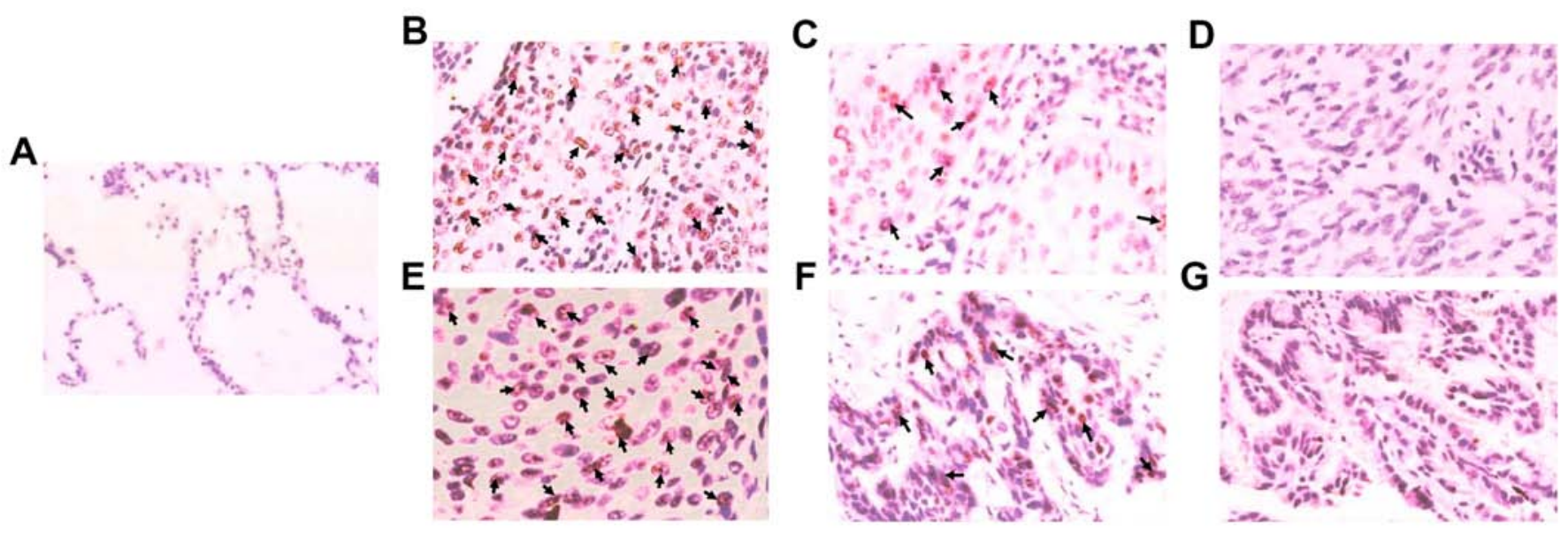

Figure 1. Representative images of histology. (A) Negative expression of MTA1 protein in para-carcinoma normal tissues (magnification, x200). (B) High expression of MTA1 protein in lung squamous carcinoma (magnification, x400). (C) Low expression of MTA1 protein in lung squamous carcinoma (magnification, x400). (D) Negative expression of MTA1 protein in lung squamous carcinoma (magnification, x400). (E) High expression of MTA1 protein in lung adenocarcinoma (magnification, x400). (F) Low expression of MTA1 protein in lung adenocarcinoma (magnification, $x 400)$. (G) Negative expression of MTA1 protein in lung adenocarcinoma (magnification, $\mathrm{x} 400$ ).

Table II. Association between MTA1 and clinicopathological features of patients [n (\%)].

\begin{tabular}{|c|c|c|c|c|c|}
\hline Item & No. & High expression MTA1 & Low expression MTA1 & $\chi^{2}$ & P-value \\
\hline \multicolumn{6}{|l|}{ Age, years } \\
\hline$<75$ & 55 & $33(60.00)$ & $22(40.00)$ & \multirow[t]{2}{*}{0.001} & \multirow[t]{2}{*}{0.983} \\
\hline$\geq 75$ & 43 & $25(58.14)$ & $18(41.86)$ & & \\
\hline \multicolumn{6}{|l|}{ Sex } \\
\hline Male & 69 & $41(59.42)$ & $28(40.58)$ & \multirow{2}{*}{0.023} & \multirow[t]{2}{*}{0.879} \\
\hline Female & 29 & $17(58.62)$ & $12(41.38)$ & & \\
\hline \multicolumn{6}{|l|}{ Tissue type } \\
\hline Squamous carcinoma & 53 & $31(58.49)$ & $22(41.51)$ & \multirow[t]{2}{*}{0.001} & \multirow[t]{2}{*}{0.954} \\
\hline Adenocarcinoma & 45 & $27(60.00)$ & $18(40.00)$ & & \\
\hline \multicolumn{6}{|l|}{ Degree of differentiation } \\
\hline Well differentiated & 23 & $6(26.09)$ & $17(73.91)$ & \multirow[t]{3}{*}{24.262} & \multirow[t]{3}{*}{$<0.001$} \\
\hline Moderately differentiated & 30 & $14(46.67)$ & $16(53.33)$ & & \\
\hline Poorly differentiated & 45 & $38(84.44)$ & $7(15.56)$ & & \\
\hline \multicolumn{6}{|l|}{ Lymph node metastasis } \\
\hline+ & 63 & $51(80.95)$ & $12(19.05)$ & \multirow[t]{2}{*}{32.127} & \multirow[t]{2}{*}{$<0.001$} \\
\hline- & 35 & $7(20.00)$ & $28(80.00)$ & & \\
\hline \multicolumn{6}{|l|}{ Clinical stage } \\
\hline I-II & 34 & $9(26.47)$ & $25(73.53)$ & \multirow[t]{2}{*}{21.037} & \multirow[t]{2}{*}{$<0.001$} \\
\hline III-IV & 64 & $49(76.56)$ & $15(23.44)$ & & \\
\hline
\end{tabular}

Association between $C T$ perfusion parameters and MTAl expression. There were no obvious differences in BV,BF and TTP between MTA1 high expression group and MTA1 low expression group. PMB was higher in MTA1 high expression group than that in MTA1 low expression group $(\mathrm{P}<0.05)$ (Table IV).

Survival status of patients with different MTAl expression. Patients with low expression of MTA1 had significantly longer survival time and a remarkably higher 5-year survival rate than those with high expression of MTA1 $(\mathrm{P}<0.05)$ (Table V).

\section{Discussion}

NSCLC is a malignant tumor originating from the bronchial mucous epithelium, its pathogenesis is not fully clear. It is generally believed that smoking, occupational carcinogens, air 
Table III. MTA1 expression in patients with different CT image features [n (\%)].

\begin{tabular}{lccccr}
\hline CT image features & No. & High expression MTA1 & Low expression MTA1 & $\chi^{2}$ & P-value \\
\hline Lobulation sign & 87 & $51(58.62)$ & $36(41.38)$ & 0.001 & 0.994 \\
No lobulation sign & 11 & $7(63.64)$ & $4(36.36)$ & & \\
Spicule sign & 60 & $50(83.33)$ & $10(16.67)$ & 34.824 & 0.983 \\
No spicule sign & 38 & $8(21.05)$ & $30(78.95)$ & & \\
Pleural indentation sign & 61 & $52(85.25)$ & $31(83.78)$ & 42.617 & 0.983 \\
No pleural indentation sign & 37 & $6(16.22)$ & $12(19.05)$ & 21.037 & $<0.001$ \\
Lymph node metastasis sign & 63 & $51(80.95)$ & $28(80.00)$ & &
\end{tabular}

CT, computed tomography; MTA1, metastasis-associated protein 1.

Table IV. Comparison of CT perfusion parameters in patients with different MTA1 expression.

\begin{tabular}{lccccc}
\hline Groups & No. & $\mathrm{BF}\left(\mathrm{ml} \bullet \mathrm{min}^{-1} \bullet 100 \mathrm{~g}^{-1}\right)$ & $\mathrm{BV}\left(\mathrm{ml} \bullet 100 \mathrm{~g}^{-1}\right)$ & $\mathrm{TTP}(\mathrm{sec})$ & $\mathrm{PMB}\left(\mathrm{ml}^{\circ} \mathrm{min}^{-1} \bullet 100 \mathrm{~g}^{-1}\right)$ \\
\hline Low expression MTA1 & 40 & $27.84 \pm 3.05$ & $6.38 \pm 1.72$ & $24.78 \pm 3.14$ & $17.52 \pm 3.65$ \\
High expression MTA1 & 58 & $28.18 \pm 3.52$ & $6.59 \pm 1.86$ & $25.53 \pm 3.02$ & $32.48 \pm 3.15$ \\
t-test & & 0.41 & 0.28 & 0.86 & 33.512 \\
P-value & $>0.05$ & $>0.05$ & $>0.05$ & $<0.001$ \\
\hline
\end{tabular}

CT, computed tomography; MTA1, metastasis-associated protein 1; BF, blood flow; BV, blood volume; TTP, time to peak; PMB permeability.

Table V. Comparison of 5-year follow-up conditions between the groups.

\begin{tabular}{lccc}
\hline Groups & No. & 5-year survival [n (\%)] & Mean survival time (months) \\
\hline Low expression MTA1 & 40 & $26(65.00)$ & $59.85 \pm 7.38$ \\
High expression MTA1 & 58 & $24(41.38)$ & $44.52 \pm 6.63$ \\
$\chi^{2 / t}$ & & 4.383 & 9.614 \\
P-value & & 0.036 & $<0.001$ \\
\hline
\end{tabular}

MTA1, metastasis-associated protein 1.

pollution, ionizing radiation, diet and nutrition, hereditary and genetic changes are the major precipitating factors, under the influence of which the carcinogens will cause damage to lung tissues. Due to the low immunity of the elderly, it is difficult for the self-defense and scavenging system of the body's immune mechanism to completely eliminate these carcinogens, so that the inflammatory response is induced, and abnormal changes are caused in the microenvironment of lungs, ultimately leading to the formation of malignant tumors (13-15). Due to atypical early symptoms, NSCLC is usually diagnosed in the middle and advanced stage, during which the tumor is prone to metastasis and patients have poor prognosis (16).

MTA1 is a recently discovered protein associated with tumor metastasis, and it belongs to the tumor metastasisassociated protein family (17). Related studies have confirmed that the expression level of MTA1 is extremely low in normal tissues but obviously high in malignant tumor tissues, and it has a close association with tumor metastasis (18). In this study, the positive expression rate of MTA1 in 98 patients was $60.20 \%$ (59/98), and MTA1 was mainly expressed in the nucleus of carcinoma tissues. The positive expression of MTA1 was higher in poorly differentiated carcinoma than that in moderately differentiated and well differentiated carcinoma, and the MTA1 expression was very low in para-carcinoma normal tissues. This is because MTA1 can be detected in the nucleus of cancer tissues due to its high hydrophilicity and no transmembrane feature. In this study, the expression of MTA1 was associated with the degree of differentiation, stage and lymph node metastasis $(\mathrm{P}<0.05)$, but not with age, gender or tissue type $(\mathrm{P}>0.05)$. The reason is that MTA1 can inhibit ER gene transcription, thereby promoting down-regulation of ER expression, and facilitating the proliferation, invasion and metastasis of tumor cells, which make the prognosis of patients poorer and shorten their survival time. 
CT examination has important value in the clinical diagnosis and treatment of NSCLC, characterized by clear images and short scanning time, and it is not influenced by surrounding tissues and organs (19). CT examination can be used to accurately observe the mass of NSCLC and to assess whether there is enlargement of lymph nodes and metastasis (20). With the development of imaging genomics, the NSCLC tissues can be quantitatively analyzed, so that the images can be converted into researchable data to reveal the intrinsic relation between tumor imaging features and molecular markers, which is of positive significance in predicting related gene mutations, and can offer strong support to targeted therapy of NSCLC (21). In this study, there was overexpression of MTA1 in tumor tissues in elderly NSCLC patients with spicule sign, pleural indentation sign and lymph node metastasis sign in CT images, indicating that patients with high expression of MTA1 are more prone to spicule sign, pleural indentation sign and lymph node metastasis sign. CT perfusion imaging technique can continuously scan the lesion area after high-pressure intravenous injection of contrast agent, thereby reflecting the hemodynamics of tumor tissues $(22,23)$. In this study, there were no obvious differences in BV, BF and TTP between high expression MTA1 group and low expression MTA1 group. PMB was higher in high expression MTA1 group than that in low expression MTA1 group $(\mathrm{P}<0.05)$. The possible reason is that the overexpression of MTA1 will regulate the cell transcription level and affects tumor angiogenesis, but such a pro-angiogenic effect is not significant enough to alter the CT perfusion parameters BV, BF and TTP. However, in the case of MTA1 overexpression, the tumor is more prone to hematogenous metastasis, and PMB is obviously increased. As far as we know, MAT1 can only be detected in tissue, but not in the blood. In clinic, the lung tissues of patients were not always acquired for some reasons while $\mathrm{CT}$ examination was easy to obtain. The results of this study provide more evidence for targeted therapy of elderly NSCLC.

In conclusion, the expression of MTA1 has close associations with the CT features, pathology and prognosis of elderly NSCLC patients, which has positive guiding significance for targeted therapy of elderly with NSCLC.

\section{Acknowledgements}

Not applicable.

\section{Funding}

No funding was received.

\section{Availability of data and materials}

All data generated or analyzed during this study are included in this published article.

\section{Authors' contributions}

NY, CL and JH designed the study and performed the experiments. NY, CL and XH acquired the data. $\mathrm{ZF}, \mathrm{XH}$ and $\mathrm{FQ}$ analyzed the data. NY, CL and JH wrote the manuscript. All authors read and approved the final manuscript.

\section{Ethics approval and consent to participate}

The study was approved by the Ethics Committee of Shandong Provincial Hospital (Shandong, China). Written informed consents were obtained from the patients and/or guardians.

\section{Patient consent for publication}

Not applicable.

\section{Competing interests}

The authors declare that they have no competing interests.

\section{References}

1. Jamal-Hanjani M, Wilson GA, McGranahan N, Birkbak NJ, Watkins TBK, Veeriah S, Shafi S, Johnson DH, Mitter R, Rosenthal R, et al; TRACERx Consortium: Tracking the evolution of non-small-cell lung cancer. N Engl J Med 376: 2109-2121, 2017.

2. Liu L, Zhou XY, Zhang JQ, Wang GG, He J, Chen YY, Huang C, Li L and Li SQ: LncRNA HULC promotes non-small cell lung cancer cell proliferation and inhibits the apoptosis by up-regulating sphingosine kinase 1 (SPHK1) and its downstream PI3K/ Akt pathway. Eur Rev Med Pharmacol Sci 22: 8722-8730, 2018.

3. Peters S, Camidge DR, Shaw AT, Gadgeel S, Ahn JS, Kim DW, Ou SI, Pérol M, Dziadziuszko R, Rosell R, et al; ALEX Trial Investigators: Alectinib versus crizotinib in untreated ALK-positive non-small-cell lung cancer. N Engl J Med 377: 829-838, 2017.

4. Rotow $\mathrm{J}$ and Bivona TG: Understanding and targeting resistance mechanisms in NSCLC. Nat Rev Cancer 17: 637-658, 2017.

5. Hendriks LEL and Dingemans AC: Heat shock protein antagonists in early stage clinical trials for NSCLC. Expert Opin Investig Drugs 26: 541-550, 2017.

6. Ma K, Fan Y, Dong X, Dong D, Guo Y, Wei X, Ning J, Geng Q, Wang $\mathrm{C}, \mathrm{Hu} \mathrm{Y}$, et al: MTA1 promotes epithelial to mesenchymal transition and metastasis in non-small-cell lung cancer. Oncotarget 8: 38825-38840, 2017.

7. Zhu X, Guo Y, Li X, Ding Y and Chen L: Metastasis-associated protein 1 nuclear expression is associated with tumor progression and clinical outcome in patients with non-small cell lung cancer. J Thorac Oncol 5: 1159-1166, 2010.

8. Hofer MD, Tapia C, Browne TJ, Mirlacher M, Sauter G and Rubin MA: Comprehensive analysis of the expression of the metastasis-associated gene 1 in human neoplastic tissue. Arch Pathol Lab Med 130: 989-996, 2006.

9. Sasaki H, Moriyama S, Nakashima Y, Kobayashi Y, Yukiue H, Kaji M, Fukai I, Kiriyama M, Yamakawa Y and Fujii Y: Expression of the MTA1 mRNA in advanced lung cancer. Lung Cancer 35: 149-154, 2002.

10. Swensen SJ: CT screening for lung cancer. AJR Am J Roentgenol 179: 833-836, 2002.

11. Goss G, Tsai CM, Shepherd FA, Ahn MJ, Bazhenova L, Crinò L, de Marinis F, Felip E, Morabito A, Hodge R, et al: CNS response to osimertinib in patients with T790M-positive advanced NSCLC: Pooled data from two phase II trials. Ann Oncol 29: 687-693, 2018.

12. Jagadish N, Parashar D, Gupta N, Agarwal S, Sharma A, Fatima R, Suri V, Kumar R, Gupta A, Lohiya NK, et al: A novel cancer testis antigen target A-kinase anchor protein (AKAP4) for the early diagnosis and immunotherapy of colon cancer. OncoImmunology 5: e1078965, 2016.

13. Shi Y, Sun Y, Yu J, Ding C, Wang Z, Wang C, Wang D, Wang C, Wang Z, Wang M, et al: China experts consensus on the diagnosis and treatment of advanced stage primary lung cancer (2016 Version) (In Chinese).

14. Cui Y, Zhang F, Zhu C, Geng L, Tian T and Liu H: Upregulated lncRNA SNHG1 contributes to progression of non-small cell lung cancer through inhibition of miR-101-3p and activation of Wnt/3catenin signaling pathway. Oncotarget 8: 17785-17794, 2017.

15. Wang Q, Wang Q, Wang SF, Jiao LJ, Zhang RX, Zhong Y, Zhang J and Xu L: Oral Chinese herbal medicine as maintenance treatment after chemotherapy for advanced non-small-cell lung cancer: A systematic review and meta-analysis. Curr Oncol 24: 269-276, 2017. 
16. Vesel M, Rapp J, Feller D, Kiss E, Jaromi L, Meggyes M, Miskei G, Duga B, Smuk G, Laszlo T, et al: ABCB1 and ABCG2 drug transporters are differentially expressed in non-small cell lung cancers (NSCLC) and expression is modified by cisplatin treatment via altered Wnt signaling. Respir Res 18: 52, 2017.

17. Jia QP, Yan CY, Zheng XR, Pan X, Cao X and Cao L: Upregulation of MTA1 expression by human papillomavirus infection promotes CDDP resistance in cervical cancer cells via modulation of NF- $\mathrm{B}$ /APOBEC3B cascade. Cancer Chemother Pharmacol 83: 625-637, 2019.

18. Bui-Nguyen TM, Pakala SB, Sirigiri DR, Martin E, Murad F and Kumar R: Stimulation of inducible nitric oxide by hepatitis B virus transactivator protein-HBx requires MTA1 coregulator. J Biol Chem 292: 4765, 2017.

19. Li HS, Lv RQ and Liu L: Correlation of CT indicators of NSCLC and pathological features and the expression level of p53 and c-myc. Eur Rev Med Pharmacol Sci 22: 135-141, 2018.
20. Lee J, Cui Y, Sun X, Li B, Wu J, Li D, Gensheimer MF, Loo BW Jr, Diehn M and Li R: Prognostic value and molecular correlates of a CT image-based quantitative pleural contact index in early stage NSCLC. Eur Radiol 28: 736-746, 2018.

21. Abel S, Hasan S, White R, Schumacher L, Finley G, Colonias A and Wegner RE: Stereotactic ablative radiotherapy (SABR) in early stage non-small cell lung cancer: Comparing survival outcomes in adenocarcinoma and squamous cell carcinoma. Lung Cancer 128: 127-133, 2019.

22. Carrascosa P and Capunay C: Myocardial CT perfusion imaging for ischemia detection. Cardiovasc Diagn Ther 7: 112-128, 2017.

23. Chu LL, Knebel RJ, Shay AD, Santos J, Badawi RD, Gandara DR and Knollmann FD: CT perfusion imaging of lung cancer: Benefit of motion correction for blood flow estimates. Eur Radiol 28: 5069-5075, 2018. 